\title{
Panoramic Spectroscopy of Sy Galaxies with elongated radio structures
}

\author{
A. A. Smirnova and A. V. Moiseev \\ Special Astrophysical Observatory, Russian Academy of Sciences, 369167 Russia \\ email: alexiya@sao.ru
}

\begin{abstract}
We have studied the ionized gas and stellar kinematics in several galaxies with active nuclei and elongated radio structures using 3D spectroscopy. The observations were performed at the SAO RAS 6-m telescope with the integral-field spectrograph MPFS (Afanasiev et al. 2001) and with a scanning Fabry-Perot interferometer (FPI) in the multimode device SCORPIO (Afanasiev \& Moiseev 2005). Based on these data the intensity maps and velocity fields in the different emission lines of the ionizing gas were constructed. Using the lines-ratio diagrams we tried to search a source of the gas ionization: an active nucleus, hot young stars or shock waves.
\end{abstract}

Keywords. galaxies: Seyfert, galaxies: jets, galaxies: kinematics and dynamics

\section{Mrk 334 (Sy1.8)}

Velocity fields in the $[\mathrm{OIII}] \lambda 4959,5007 \AA$ emission lines shows a significant (150$200 \mathrm{~km} / \mathrm{s}$ ) 'blue' excess of the line-of-sight velocities in the galactic nucleus. This feature seems like an outflow from AGN caused by the circumnuclear radio structure (Kukula et al. 1995). The ionized gas velocities in the other emission lines show almost circular rotation. However, the maps of deviations from the circular model reveals large-scale non-circular motions near two bright emission-line condensations (we call it ' $k$ not A' and ' $k$ not B'). The non-circular motions is about $-50 \mathrm{~km} / \mathrm{s}$ near the knot B and exceed +60 $\mathrm{km} / \mathrm{s}$ between knots $\mathrm{A}$ and $\mathrm{B}$. These peculiar streaming motions may be connect with the process of an interaction (merging) with a companion.

\section{Mrk 1066 (Sy2)}

The MPFS and FPI velocity fields in the different emission lines were analyzed by means the 'tilted-rings' methods. Most emission lines (except oxygen ones) exhibit a regular circular rotation. Line-of-sight velocities in the $[\mathrm{OII}] \lambda 3727 \AA$ and $[\mathrm{OIII}] \lambda 4959$, $5007 \AA$ show significant deviations from the model of pure circular rotation. The main fraction of the [OIII] velocities are non-circular. This effect is a possible combination of two kinematically different subsystems: a normal rotation and an outflow caused by the radio jet intrusion in an ambient medium.

\section{Acknowledgements}

This work was supported by the RFBR grant 06-02-16825.

\section{References}

Afanasiev, V.L., Dodonov, S.N. \& Moiseev, A.V. 2001, in: L.P. Osipkov \& I.I. Nikiforov (eds.), Stellar dynamics: from classic to modern, p.103.

Afanasiev, V.L. \& Moiseev, A.V., 2005, Astron. Letters, 31, 193, astro-ph/0502095.

Kukula, M.J., Pedlar, A., Baum, S.A., \& O’Dea, C. P. 1995, MNRAS, 276, 1262. 